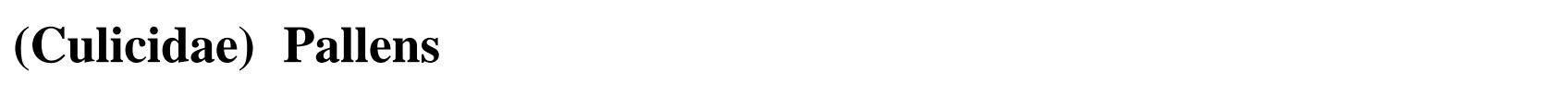 Culex pipiens واليرقلت غير المستهفة للهاdوث (Chironomidae)
}

\section{Chironomus ninevah Ahmad}

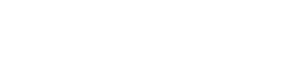 \\ قنم علوم الحية \\ كلية التربية \\ جلمعة الموصل
}

Email: dr_atallah1957@yahoo.com

(تاريخ الاستلام 10 / /2011 ؛ تاريخ القبول 26 /12 / 2011)

\section{الملغص}

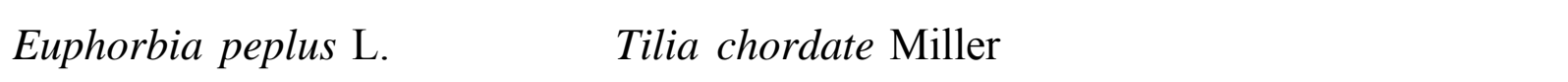

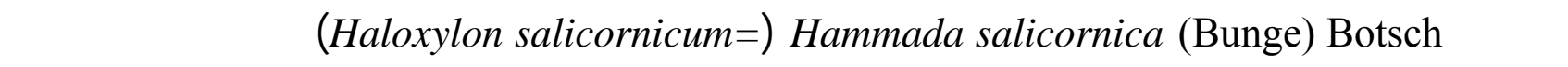
ضد لُطوار بعوض الكيولكس Solanum carolinense L. كلت قيم Lcex pipiens Pallens.

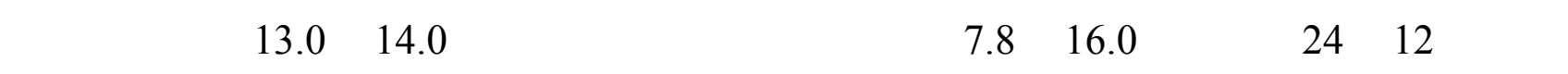

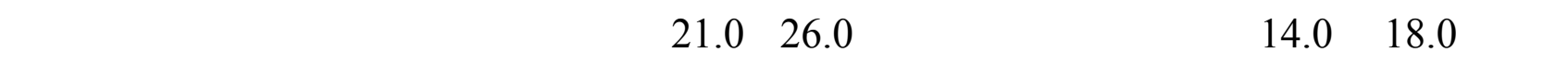

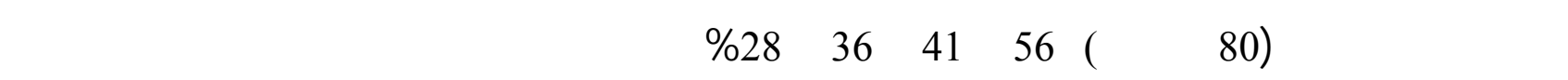

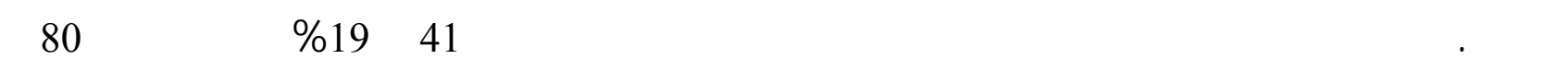

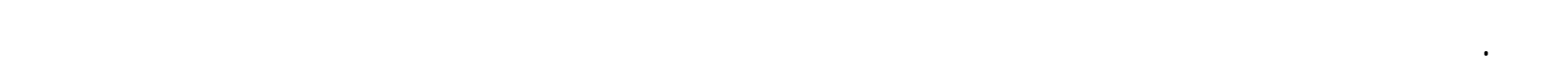
Chironomus ninevah Ahmad والطمطة البرية والرمث على التوالي.

الهاملت الدالة: Culex pipiens، Chironomus ninevah، مستخلصك نباتية، نسبة القل. 
عالمفردمخلف

\title{
Bioefficacy of Four Botanical Extracts Against Culex pipiens Pallens (Culicidae) Stages and Non-Targeted Chironomus ninevah Ahmad (Chironomidae)
}

\author{
Atallah F. Mekhlif \\ Department of Biology \\ College of Education \\ University of Mosul \\ Email: dr atallah1957@yahoo.com
}

\begin{abstract}
Bioinsecticidal activity of Tilia chordate Miller, Euphorbia peplus L., Solanum carolinense L. and Hammada salicornica (Bunge) Botsch (=Haloxylon salicornicum) were tested against various stages of Culex pipiens Pallens and Chironomus ninevah Ahmad $4^{\text {th }}$ larvae. For 12 and 24 hrs. exposure times, $\operatorname{Lc}_{50}$ were $(16.0,7.5 \mathrm{ppm}),(14.0,13.0 \mathrm{ppm})$, $(18.0,26.0 \mathrm{ppm})$ and $(26.0,21.0 \mathrm{ppm})$ caused by T. chordate, E. peplus, $H$. salicornica and S. carolinense respectively. Pupal mortality were 56, 41,36 and 28\% for T. chordata, $H$. salicornica, S. carolinense, and E. peplus at the later $(80 \mathrm{ppm})$ applied concentration. Only, $T$. chordata, and $H$. salicornica, extracts were active on adult exclusion with mortality 41 and $19 \%$ at $80 \mathrm{ppm}$ concentration.

On the other hand, its revealed that significant differences between $\mathrm{Lc}_{50}$ of the targeted C. pipiens larvae and that of the non-targeted C. ninevah larvae with 10, 4 and 2 folds of the applied extracts ; T. chordata, S. carolinense and H. salicornica.
\end{abstract}

Keywords: Culex pipiens ,Chironomus ninevah, Plant Extracts, Mortality.

\section{الفمصة}

إن لستخده المبيدك التركيبية في مكلفحة البعوض، قد ترك بقاياسلمة في المنتجلت الغذائية وليب من للسهل تحللها بيولوجياً، إضلفةً إلى خطورة تداول تلك المبيدات (Sukumar et al., 1999) ، وظهور مقاومة لها من البعوض كالنوع Culex pipiens (Zayed et al., 2006). ولكن لستخدلم المبيدات ذات الأصل النباتي يكون متخصصاً بالآفة المستهذفة بالمكلحة عموماً وغير مؤذٍ للأحياء غير المستهذفة ومن ضمنها الإن سان فهي تتحل بيولوجياً وغير ضارة للبيئة، لذك فن المعلملة بالمركبت النباتية أو إضلفتها بوصفها مـ -ؤازرات

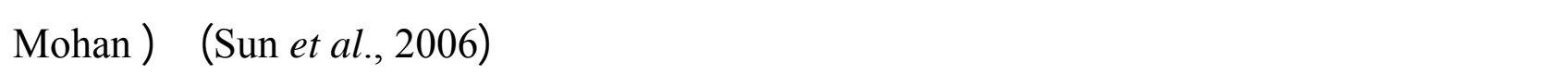
. (et al., 2010

Al-Sharook et al., ) في مجل مكلفحة البعوض بالمستخلصت ذات الأصل النباتي، فإضلفة إلى القنل

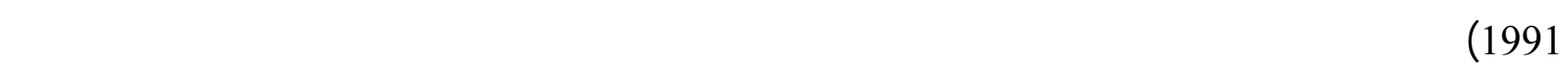

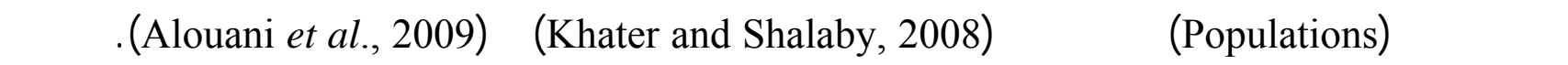




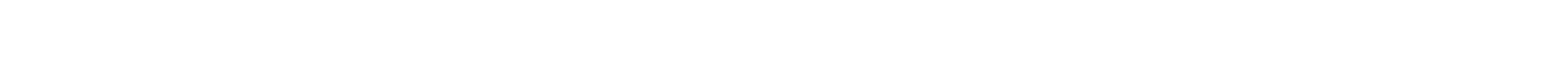

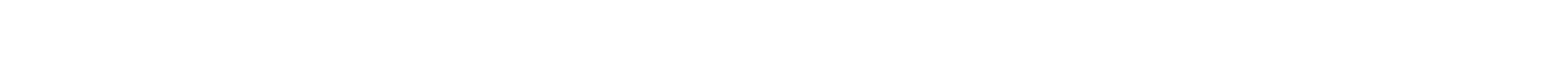
للمبيدات بالمقارنة مع مكلفحة البالغلت (El-Hag et al., 2001) ؛ (Dharmagadda et al., 2005). في حل إجراء المكافحة، فلن يرقلت جنس الهلموش .Chironomus spp تكون من ض من الأحي اء

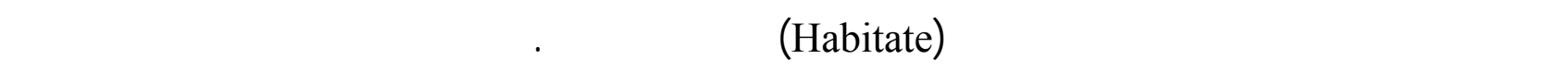

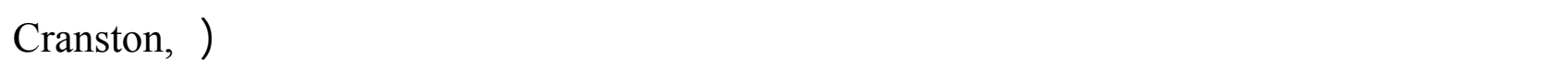
1995) و (Widwed et al., 1997) والجنات (Wais et al., 1980)

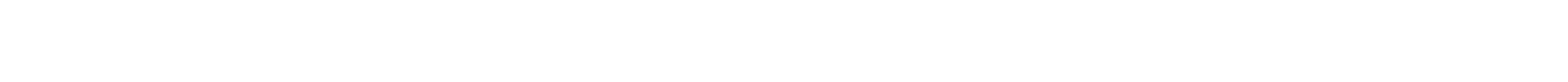

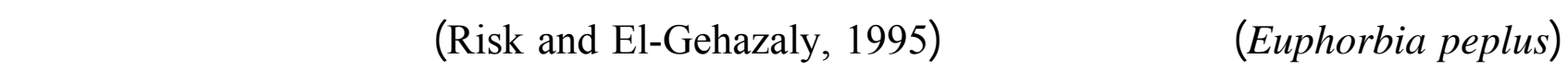

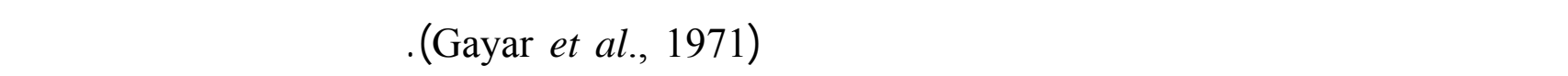
فارفا Solanum carolinense

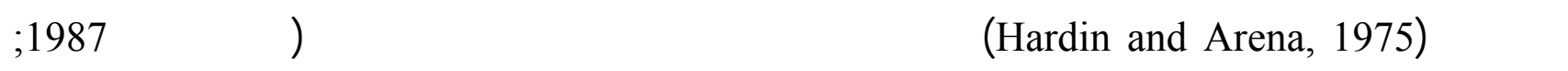

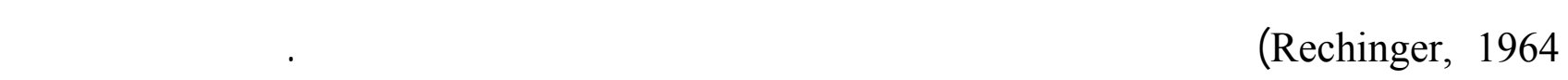
Kutas et al., أن مستخلص أوراق الزيزفون

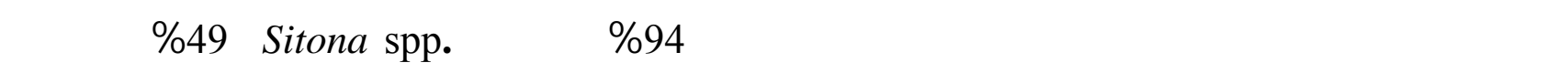

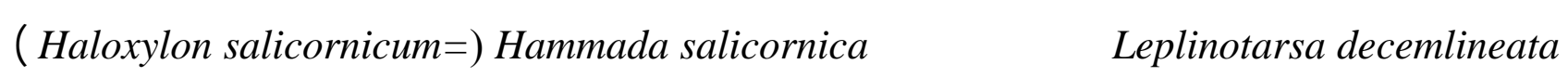
فهو نببت طبيعيسل (Al-Ahmadi and Salem, 1995). إن الهذف من هذه الدرلسة هو لختبار المستخلصت النباتية بوصفها مبيدات حيوية في مكلفحة بع -وض

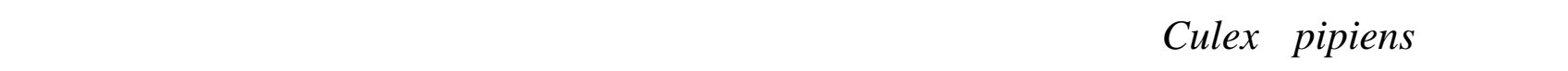

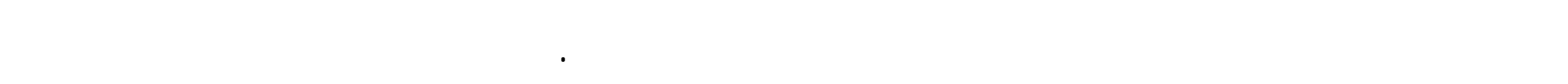
المستخمة في البعوض الهثرة المستهفة مع يرقات الهلموش Chironomidae) Chironomus ninevah غير المستهذفة وذات الموقع الرئيس فيسلسلة الغذاء في نفس بيئة البعوض C. pipiens.

\section{المواد ولرالق الهل}

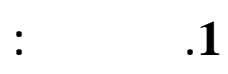

مُ جمع يرقلت البعوض Culex pipiens والهلموش Chironomus ninevah بولنطة غربل قطر

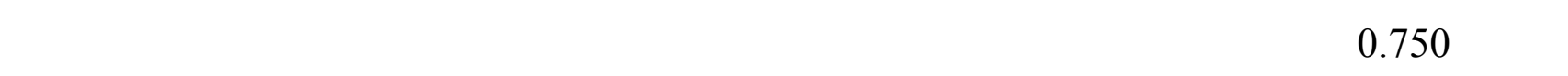

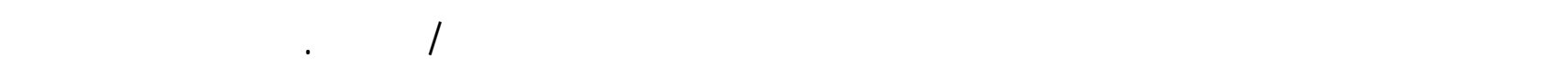


المختبر في وعاء منلهب، وقد لجريت التجارب على اليرقلت حن نخولها الطور الرايع والعذارى حل تعنيرها.

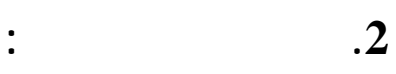

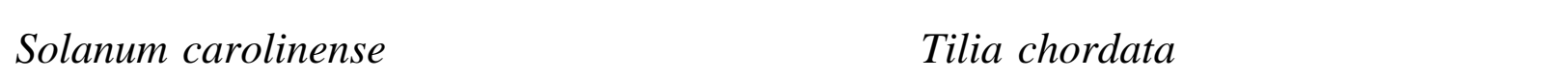

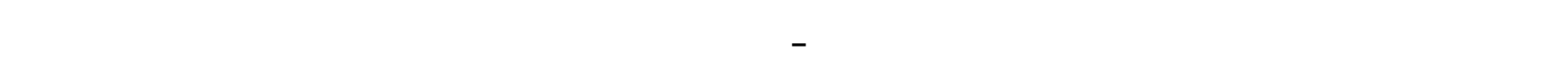

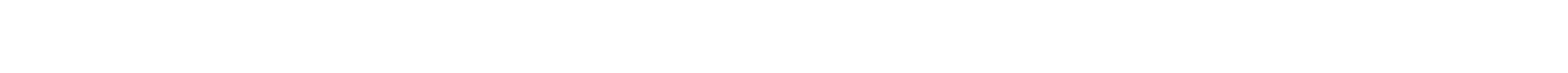
جمعت عينت لم الحليب Euphorbia peplus من صحراء الهضبة الغربية نحو 50 كم غرب مدينة القائم.

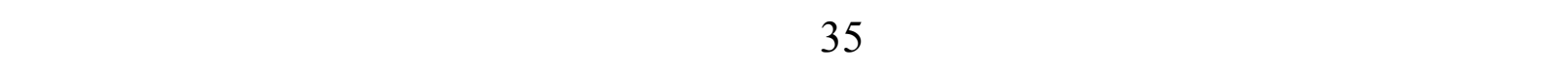

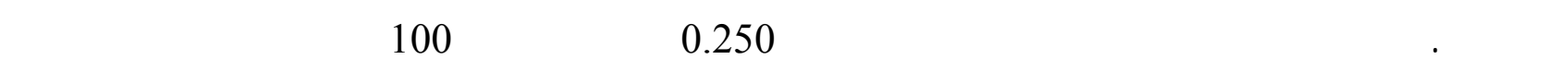

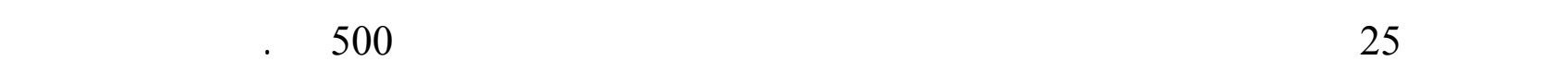

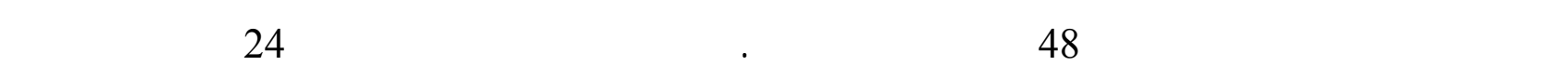

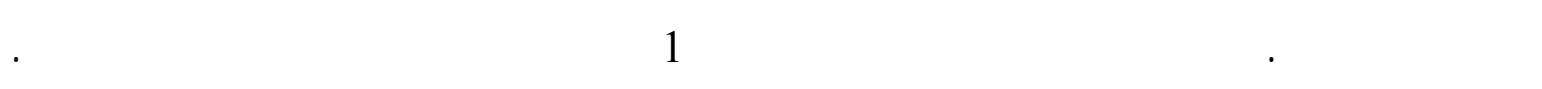
ترك الرلشحطيلة الليل لتبخير المذيب، وقد كررت إذلبة المستخلص ثانية في كحدول الايثانول، وأهمل

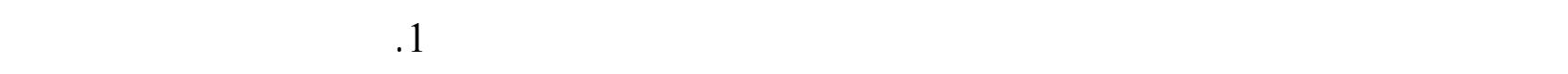

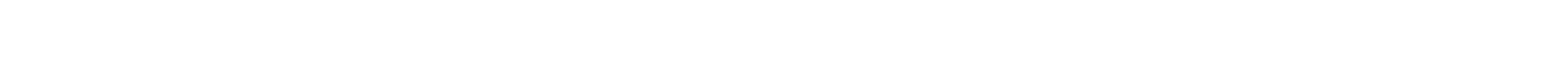

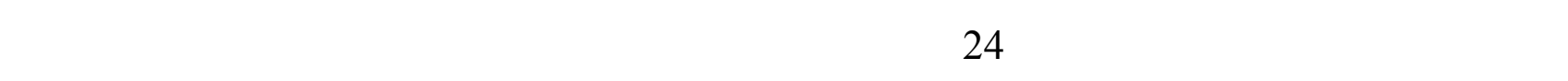

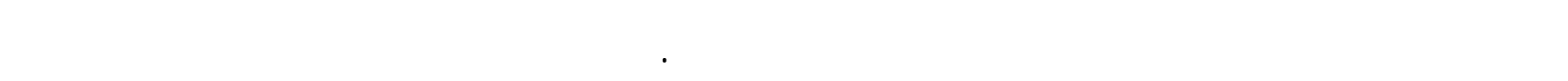

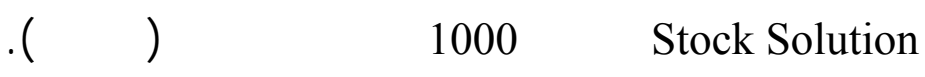

3. النجارب الأولية: جرى تحديد أعلى تركيز من مستخلص أكثر النباتلت تأثيراً على يرقلت البعوض والذي ي سبب ن سسبة

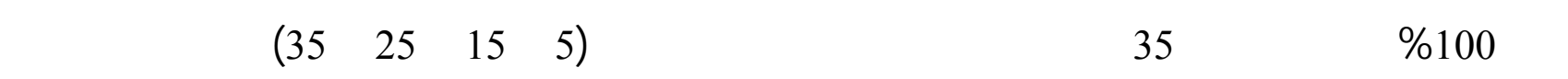

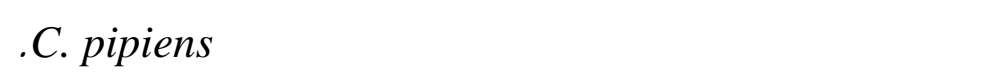

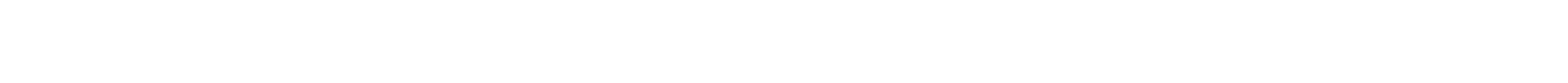

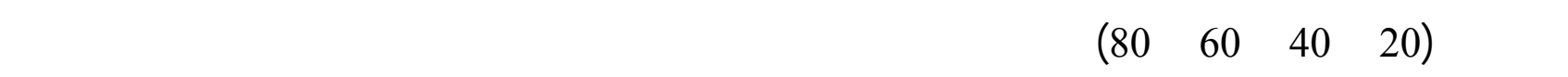

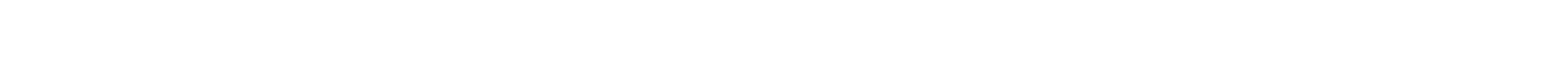
التأثير الانتخابي للمستخلصات على الطور اليرقي الرلع ليرقلت الهلموش والبعوض عدا لم الحليب.

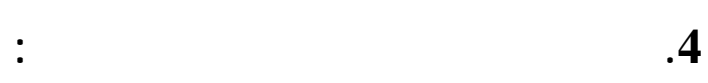

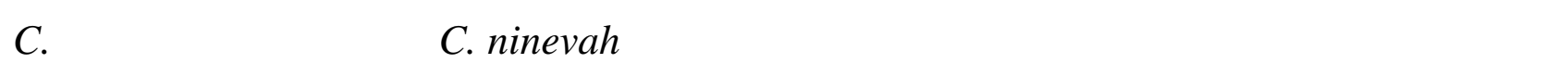

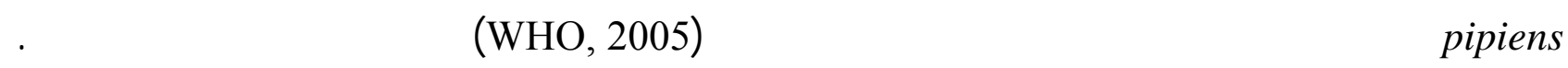


تبدأ الاختبارات بإضلفة مللتر ولحد من التركيز الذي جرى تحضيره من المحلول الأصلي إلى 100 مل من

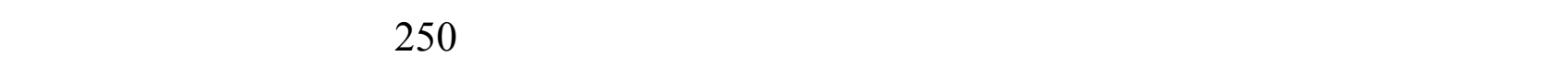

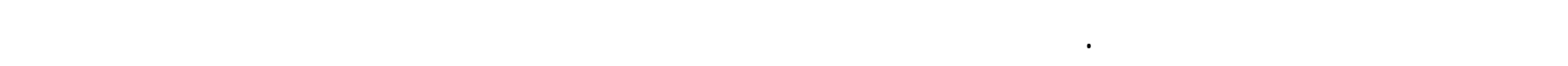
الايثانول، في حل معلملة اليرقل، يضف إلى المحلى المحلول غذاء اليرقلت والمكون من مسحوق الروبيلن الذي الذي الماء

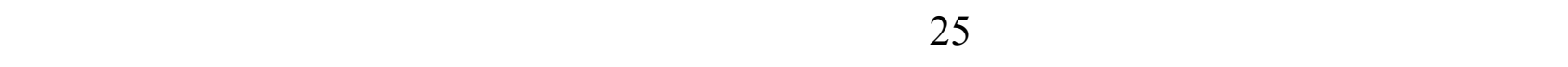
عذارى البعوض إلى الأوعية بولسطة منظل دقيق وفوشائة منلسبة.

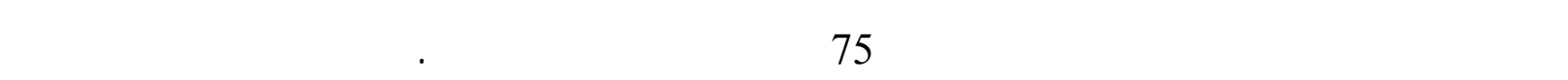

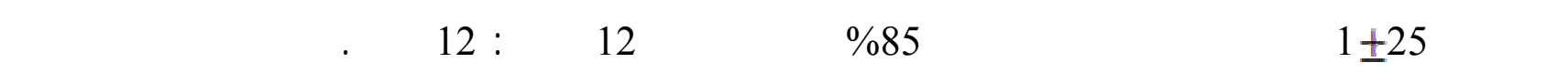

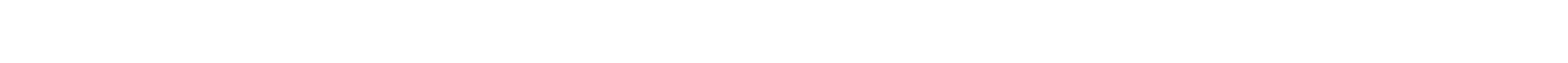

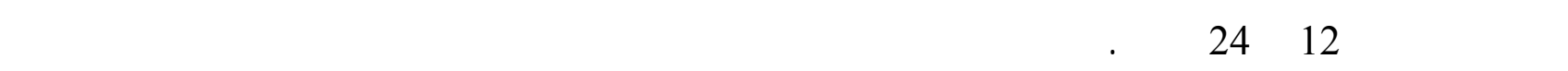

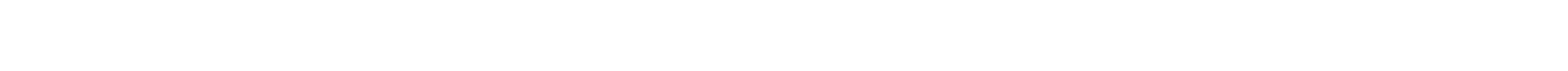

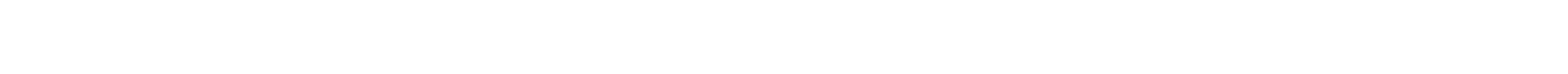

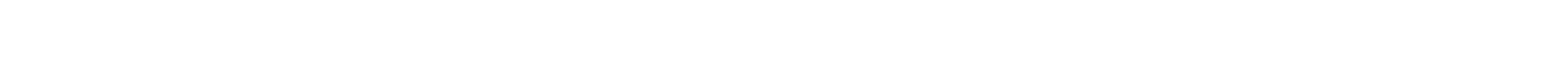
الجهات المحطة بها عاةً.

\section{النتائج والمنالثشة}

\section{1. التأثير الفالل ضذد يرقلت البعوض:}

يوضع الجدول 1 لمتجابة اليرقت للمستخلصت خلال فترقي تعريض 12 و 24ساعة. وقد لوضظ عدم

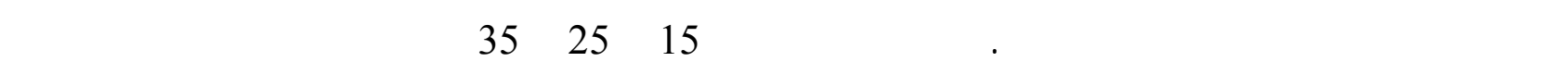

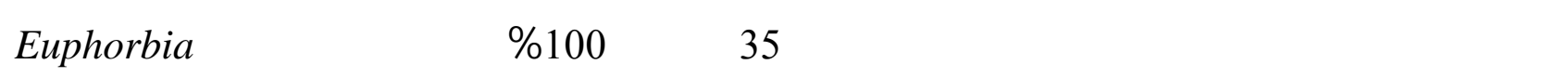
peplus

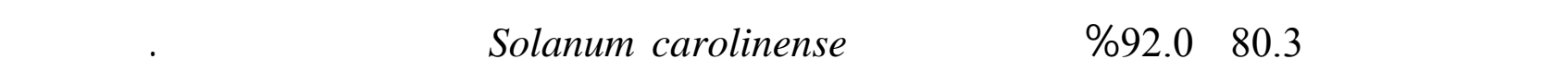
التركيز 25 ج ف م الفترة التعريض 24ساعة، فلن نسبة القل و8.7\% لمستخلص أم الحليب ويليه الزيزفون

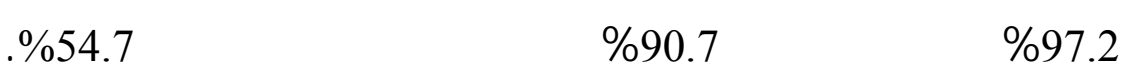

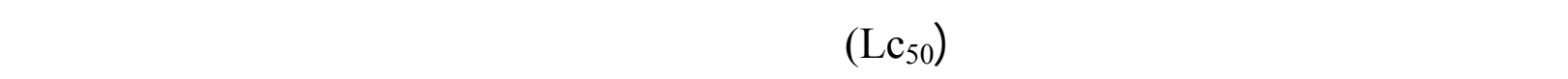

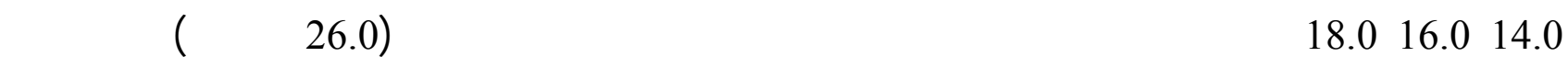

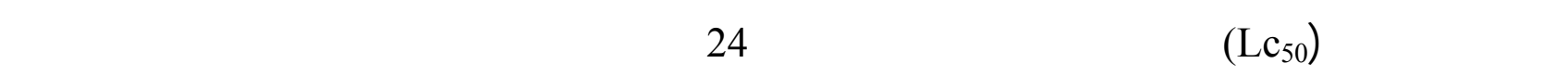

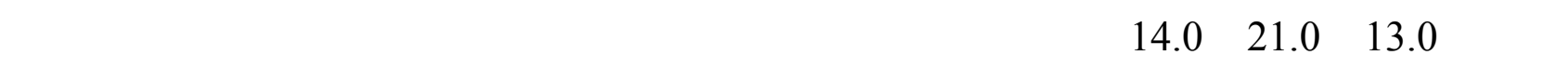
فترة التعريض 24ساعة نقريباً (جدول 1). 


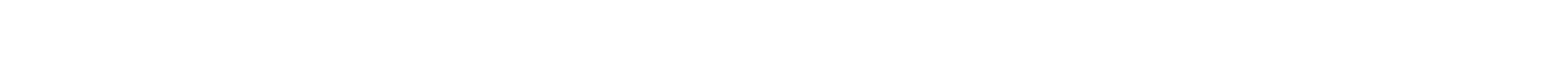

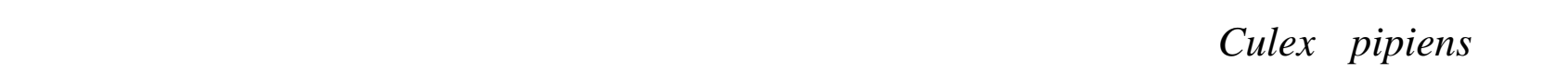

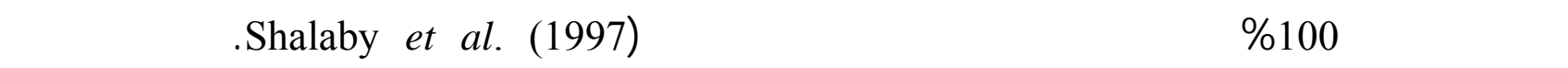
Atlantia أن مـ عستخلص كح _ول الميث انول لأوراق Siragniname and Kalyanasundrama (2004) monophylla

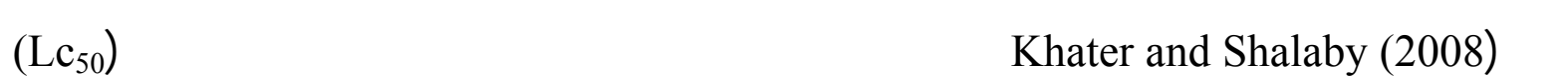

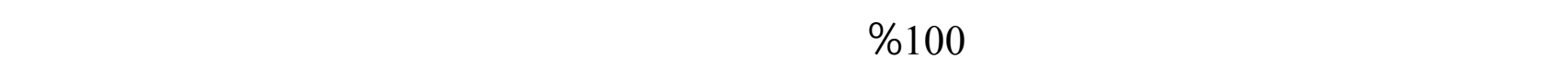
الحالية. إن النتائج الحالية تقفق مع (2009) Alouani et al., و ( Siragniname and Kalyanasundrama 2004) وJang et al., (2002 في أن للمستخلصت النباتية الخلم أو المصفلة جزئيًا رخيصة وفعالة جدأ ف مي مكافحة البعوض مقارنةً مع المركبلت القية.

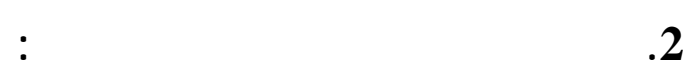
يوضح الجدول 2 عمم تأثر عذارى البعوض بإضفافة مستخلصك لم الحليب والطملطة البرية والرم ـث

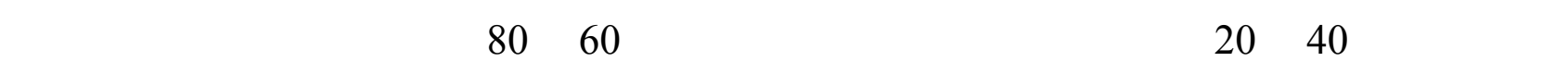

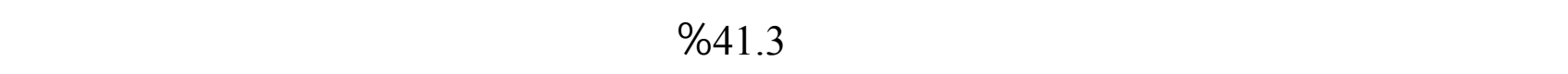

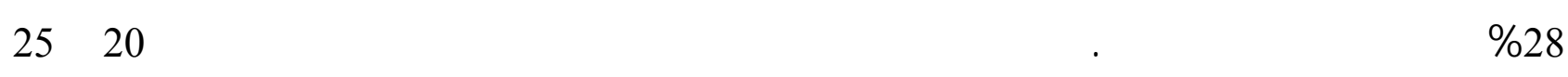
و 64 و 65\% عند الترلكيز الأربعة على التوالي.

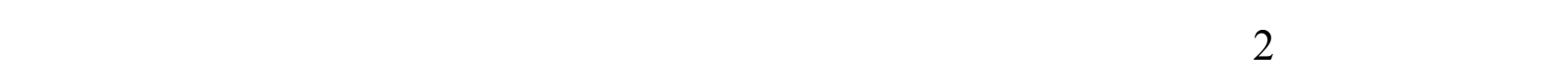

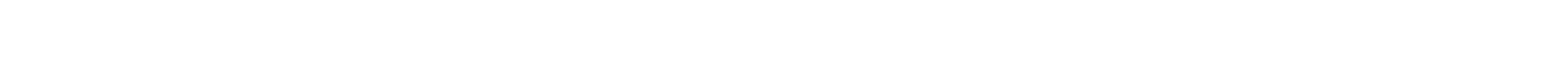

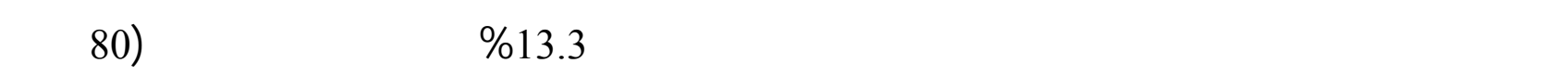

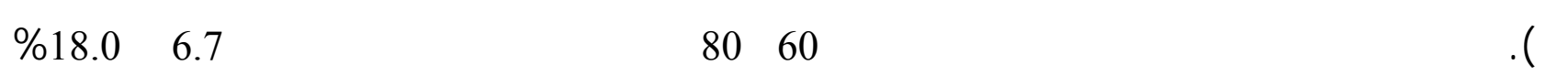

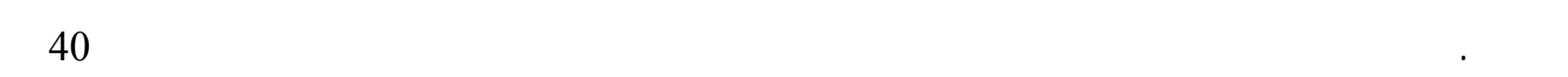

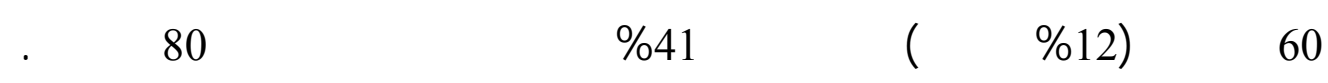
ويبين الجدول 2 أيضاًَمدى التباين في تأثير المستخلصك على على مستوى المجموع في موت العذارى وما يتبعه

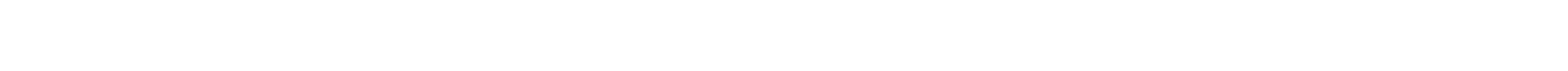

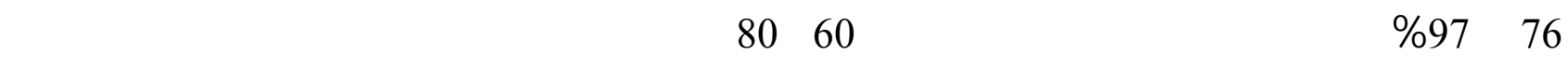

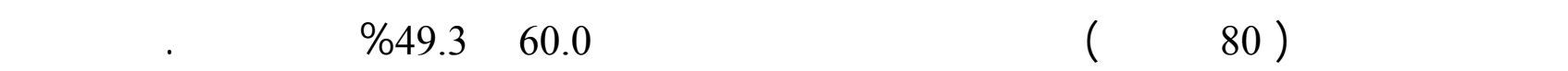

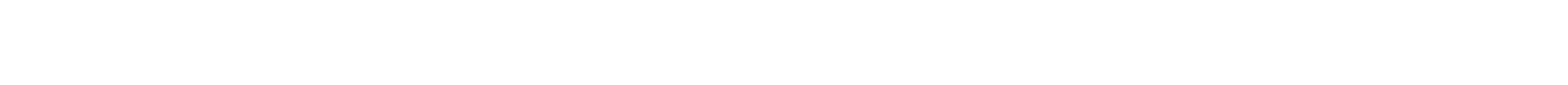
على هرمون البزوغ (Makkar et al., 2007). 
الجدول 1: تأثير المستخلصت النباتية كمبيدات حيوية في قنل يرقت الط -ور الرالب ع ليرق لت البع -وض .Culex pipiens

\begin{tabular}{|c|c|c|c|c|c|c|c|}
\hline \multirow{2}{*}{ لالحعدار } & \multirow{2}{*}{ قيمة F } & \multicolumn{2}{|c|}{ قيمة Lc } & \multicolumn{2}{|c|}{ الفل ونسبته بعدفترة التعرض } & \multirow{2}{*}{ 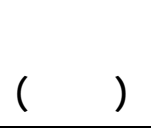 } & \multirow{2}{*}{ المستخلص } \\
\hline & & 24ساعة & 12سلساعة & 24ساعة & 12ساعة & & \\
\hline \multirow{5}{*}{$\begin{array}{l}x \\
0 \\
8 \\
0 \\
0 \\
1 \\
8 \\
8 \\
0 \\
0 \\
11 \\
\lambda\end{array}$} & \multirow{5}{*}{${ }^{* *} 71.29$} & \multirow{5}{*}{13.0} & \multirow{5}{*}{14.0} & $\begin{array}{c}0.0 \pm 0.0 \mathrm{c} \\
(0.0)\end{array}$ & $\begin{array}{c}0.0 \pm 0.0 \mathrm{c} \\
(0.0)\end{array}$ & الضاطة & \multirow{5}{*}{$\begin{array}{c}\text { له الحليب } \\
\text { E. peplus }\end{array}$} \\
\hline & & & & $\begin{array}{c}0.0 \pm 0.0 \mathrm{c} \\
(0.0)\end{array}$ & $\begin{array}{c}0.0 \pm 0.0 \mathrm{c} \\
(0.0)\end{array}$ & 5 & \\
\hline & & & & $\begin{array}{c}16.7 \pm 3.2 \mathrm{~b} \\
(66.7)\end{array}$ & $\begin{array}{c}13.7 \pm 4.0 \mathrm{~b} \\
(55.5)\end{array}$ & 15 & \\
\hline & & & & $\begin{array}{c}24.7 \pm 0.6 \mathrm{a} \\
(98.7)\end{array}$ & $\begin{array}{c}21.0 \pm 5.3 \mathrm{a} \\
(84.0)\end{array}$ & 25 & \\
\hline & & & & $\begin{array}{c}25.0 \pm 0.0 \mathrm{a} \\
(100)\end{array}$ & $\begin{array}{c}25.0 \pm 0.0 \mathrm{a} \\
(100)\end{array}$ & 35 & \\
\hline \multirow{5}{*}{ 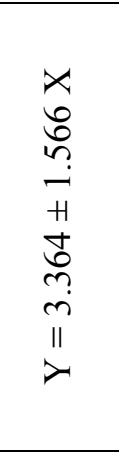 } & \multirow{5}{*}{${ }^{* *} 808.3$} & \multirow{4}{*}{7.8} & \multirow{4}{*}{16.0} & $\begin{array}{c}0.0 \pm 0.0 \mathrm{c} \\
(0.0)\end{array}$ & $\begin{array}{c}0.0 \pm 0.0 \mathrm{c} \\
(0.0)\end{array}$ & الضاطة & \multirow{5}{*}{$\begin{array}{c}\text { الزيزفون } \\
\text { T. chordate }\end{array}$} \\
\hline & & & & $\begin{array}{c}0.0 \pm 0.0 \mathrm{c} \\
(0.0)\end{array}$ & $\begin{array}{c}0.0 \pm 0.0 \mathrm{c} \\
(0.0)\end{array}$ & 5 & \\
\hline & & & & $\begin{array}{c}3.3 \pm 0.5 \mathrm{~b} \\
(13.3)\end{array}$ & $\begin{array}{c}3.3 \pm 0.5 \mathrm{~b} \\
(13.3)\end{array}$ & 15 & \\
\hline & & & & $\begin{array}{c}24.3 \pm 0.6 \mathrm{a} \\
(97.2)\end{array}$ & $\begin{array}{c}24.3 \pm 0.6 \mathrm{a} \\
(97.2)\end{array}$ & 25 & \\
\hline & & & & $\begin{array}{c}25.0 \pm 0.0 \mathrm{a} \\
(100)\end{array}$ & $\begin{array}{c}25.0 \pm 0.0 \mathrm{a} \\
(100)\end{array}$ & 35 & \\
\hline \multirow{5}{*}{ 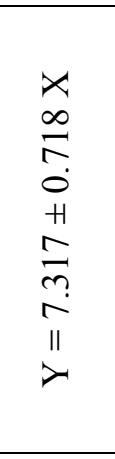 } & \multirow{5}{*}{${ }^{* *} 469.30$} & \multirow{5}{*}{14.0} & \multirow{5}{*}{18.0} & $\begin{array}{c}0.0 \pm 0.0 \mathrm{f} \\
(0.0)\end{array}$ & $\begin{array}{c}0.0 \pm 0.0 \mathrm{f} \\
(0.0)\end{array}$ & الضاطة & \multirow{5}{*}{$\begin{array}{c}\text { الرمث } \\
\text { H. } \\
\text { salicornica }\end{array}$} \\
\hline & & & & $\begin{array}{c}0.0 \pm 0.0 \mathrm{f} \\
(0.0)\end{array}$ & $\begin{array}{c}0.0 \pm 0.0 \mathrm{f} \\
(0.0)\end{array}$ & 5 & \\
\hline & & & & $\begin{array}{c}14.3 \pm 1.5 \mathrm{e} \\
(57.3)\end{array}$ & $\begin{array}{c}8.3 \pm 1.5 \mathrm{~d} \\
(33.3)\end{array}$ & 15 & \\
\hline & & & & $\begin{array}{c}22.7 \pm 0.6 \mathrm{~d} \\
(90.7)\end{array}$ & $\begin{array}{c}21.0 \pm 1.7 \mathrm{~b} \\
(84.0)\end{array}$ & 25 & \\
\hline & & & & $\begin{array}{c}25.0 \pm 0.0 \mathrm{a} \\
(100)\end{array}$ & $\begin{array}{c}25.0 \pm 0.0 \mathrm{a} \\
(100)\end{array}$ & 35 & \\
\hline \multirow{5}{*}{ 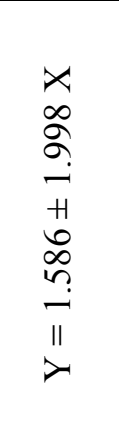 } & \multirow{5}{*}{ ** 98.89} & \multirow{5}{*}{21.0} & \multirow{5}{*}{26.0} & $\begin{array}{c}0.0 \pm 0.0 \mathrm{e} \\
(0.0)\end{array}$ & $\begin{array}{c}0.0 \pm 0.0 \mathrm{e} \\
(0.0)\end{array}$ & الضاطة & \multirow{5}{*}{$\begin{array}{c}\text { الطملة البرية } \\
\text { Carolinense } \\
\text { care }\end{array}$} \\
\hline & & & & $\begin{array}{c}0.3 \pm 0.6 \mathrm{e} \\
(1.3)\end{array}$ & $\begin{array}{c}0.0 \pm 0.0 \mathrm{e} \\
(0.0)\end{array}$ & 5 & \\
\hline & & & & $\begin{array}{c}7.0 \pm 2.0 \mathrm{c} \\
(28.0)\end{array}$ & $\begin{array}{c}3.3 \pm 0.6 \mathrm{~d} \\
(13.3)\end{array}$ & 15 & \\
\hline & & & & $\begin{array}{c}13.7 \pm 1.2 \mathrm{~b} \\
(54.7)\end{array}$ & $\begin{array}{c}11.3 \pm 3.8 \mathrm{~b} \\
(45.3)\end{array}$ & 25 & \\
\hline & & & & $\begin{array}{c}23.0 \pm 1.7 \mathrm{a} \\
(92.0)\end{array}$ & $\begin{array}{c}20.7 \pm 0.6 \mathrm{a} \\
(80.3)\end{array}$ & 35 & \\
\hline
\end{tabular}

* عدد اليرقل 25 في كل مكرر.

- لايوجد فرق معنوي بين الأرقلم الني تحمل حروفاً متشابهة في المربع (block) الولحد عندمستوى الاحتمالية 0.05 .

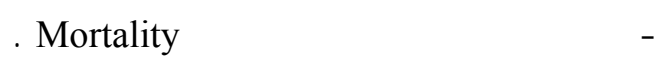


الجدول 2: تأثير المستخلصت في عذارى البعوض Culex pipiens ومغادرة الكلملات الماء.

\begin{tabular}{|c|c|c|c|c|}
\hline \multirow[b]{2}{*}{ قيمة F } & \multicolumn{2}{|c|}{ عثم الوصط إلى اللور الكلل هو } & \multirow{2}{*}{ (جفم) التركيز) } & \multirow[b]{2}{*}{ المستخلص النبالثي } \\
\hline & فلل البزوغ ومغالرتها & موت العذارى & & \\
\hline \multirow{4}{*}{$77.29^{* *}$} & $\begin{array}{c}0.0 \pm 0.0 \mathrm{c} \\
(0.0)\end{array}$ & $\begin{array}{c}0.0 \pm 0.0 \mathrm{c} \\
(0.0)\end{array}$ & 20 & \multirow{4}{*}{$\begin{array}{c}\text { لم الحليب } \\
\text { E.peplus }\end{array}$} \\
\hline & $\begin{array}{c}0.0 \pm 0.0 \mathrm{c} \\
(0.0)\end{array}$ & $\begin{array}{c}0.3 \pm 0.6 \mathrm{c} \\
(1.3)\end{array}$ & 40 & \\
\hline & $\begin{array}{c}0.0 \pm 0.0 \mathrm{c} \\
(0.0)\end{array}$ & $\begin{array}{c}5.7 \pm 1.2 \mathrm{~b} \\
(22.8)\end{array}$ & 60 & \\
\hline & $\begin{array}{c}0.0 \pm 0.0 \mathrm{c} \\
(0.0)\end{array}$ & $\begin{array}{c}7.0 \pm 1.0 \mathrm{a} \\
(28)\end{array}$ & 80 & \\
\hline \multirow{4}{*}{$82.02^{* *}$} & $\begin{array}{c}0.0 \pm 0.0 \mathrm{~d} \\
(0.0)\end{array}$ & $\begin{array}{c}0.0 \pm 0.0 \mathrm{~d} \\
(0.0)\end{array}$ & 20 & \multirow{4}{*}{$\begin{array}{c}\text { الطملة البرية } \\
\text { S. carolinense }\end{array}$} \\
\hline & $\begin{array}{c}0.0 \pm 0.0 \mathrm{~d} \\
(0.0)\end{array}$ & $\begin{array}{l}2.7 \pm 1.2 \mathrm{c} \\
(10.7)\end{array}$ & 40 & \\
\hline & $\begin{array}{c}0.0 \pm 0.0 \mathrm{~d} \\
(0.0)\end{array}$ & $\begin{array}{c}7.0 \pm 1.0 \mathrm{~b} \\
(28.0)\end{array}$ & 60 & \\
\hline & $\begin{array}{c}3.3 \pm 1.2 \mathrm{c} \\
(0.0)\end{array}$ & $\begin{array}{c}9.0 \pm 0.0 \mathrm{a} \\
(36.0)\end{array}$ & 80 & \\
\hline \multirow{4}{*}{$129.83^{* *}$} & $\begin{array}{c}0.0 \pm 0.0 \mathrm{e} \\
(0.0)\end{array}$ & $\begin{array}{c}0.0 \pm 0.0 \mathrm{e} \\
(0.0)\end{array}$ & 20 & \multirow{4}{*}{$\begin{array}{c}\text { الرمثث } \\
\text { H. salicornica }\end{array}$} \\
\hline & $\begin{array}{c}0.0 \pm 0.0 \mathrm{e} \\
(0.0)\end{array}$ & $\begin{array}{c}0.0 \pm 0.0 \mathrm{e} \\
(0.0)\end{array}$ & 40 & \\
\hline & $\begin{array}{c}1.7 \pm 0.6 \mathrm{~d} \\
(6.8)\end{array}$ & $\begin{array}{c}8.7 \pm 0.6 \mathrm{~b} \\
(34.7)\end{array}$ & 60 & \\
\hline & $\begin{array}{c}4.7 \pm 1.5 \mathrm{c} \\
(18.7) \\
\end{array}$ & $\begin{array}{c}10.3 \pm 0.6 \mathrm{a} \\
(41.3)\end{array}$ & 80 & \\
\hline \multirow{4}{*}{$64.83^{* *}$} & $\begin{array}{c}0.0 \pm 0.0 \mathrm{e} \\
(0.0)\end{array}$ & $\begin{array}{c}5.0 \pm 1.0 \mathrm{~d} \\
(20.0)\end{array}$ & 20 & \multirow{4}{*}{$\begin{array}{l}\text { الزيزفون } \\
\text { T. chordate }\end{array}$} \\
\hline & $\begin{array}{c}3.0 \pm 1.0 \mathrm{~d} \\
(12.0)\end{array}$ & $\begin{array}{c}6.3 \pm 3.1 \mathrm{c} \\
(25.0)\end{array}$ & 40 & \\
\hline & $\begin{array}{c}3.0 \pm 0.0 \mathrm{~d} \\
(12.0)\end{array}$ & $\begin{array}{c}16.0 \pm 2.0 \mathrm{a} \\
(64.0)\end{array}$ & 60 & \\
\hline & $\begin{array}{c}10.3 \pm 0.6 \mathrm{~b} \\
(41.3)\end{array}$ & $\begin{array}{c}14.0 \pm 1.0 \mathrm{a} \\
(56.0)\end{array}$ & 80 & \\
\hline
\end{tabular}

* عدد اليرقت 25 في كل مكرر.

- لايوجد فرق معنوي بين الأرقلم الني تحمل حروفأمتشابهة في المربع ( block) الولحد عند مستوى الاحتمالية 0.05 .

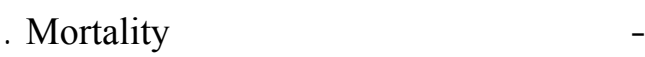


3. تأثير المستخلصك في الهاهوش:

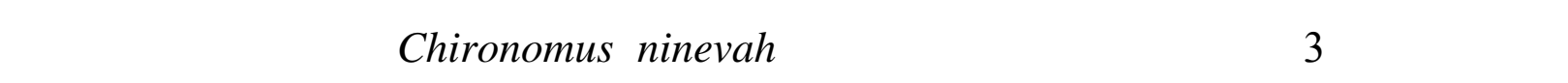

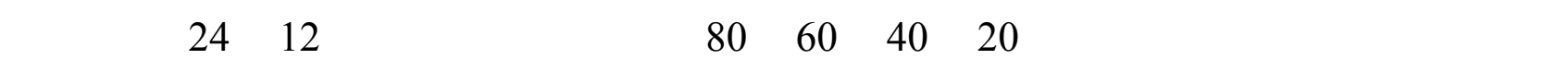

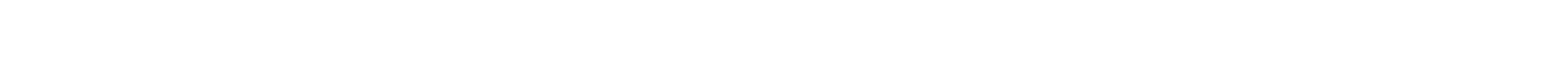

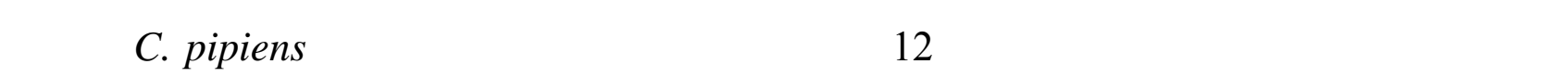

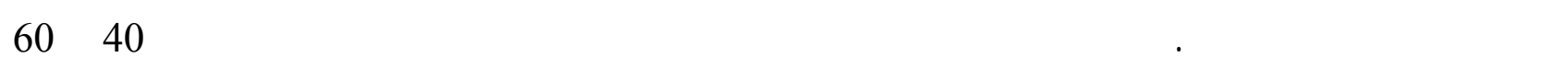

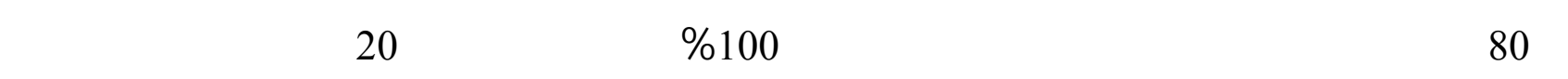
القل 100 و 26 و 20\% لمستخلصك الزيزفون والرمث والطملطة البرية على التوالي، ولم تؤوثر زيادة فترة

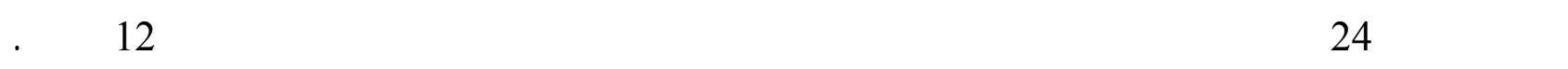

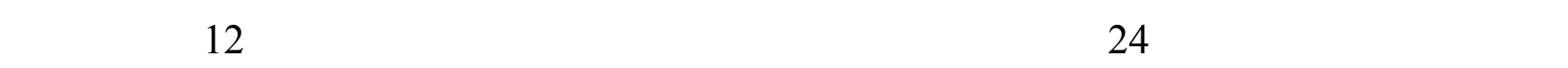

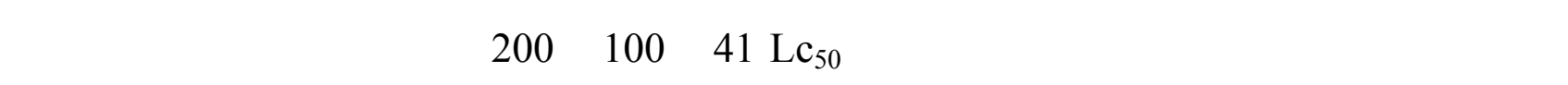
والطملة البرية والزيزفون (للشكل 1).

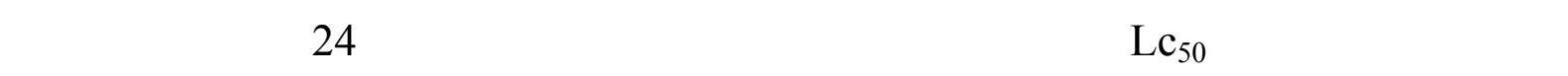

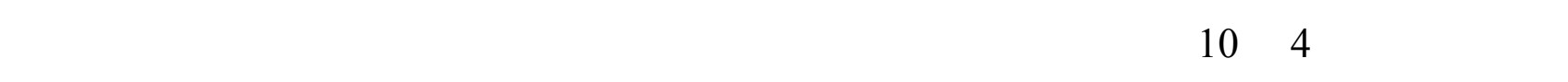

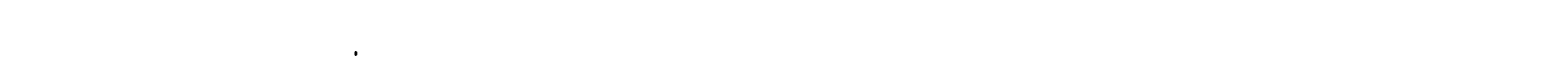

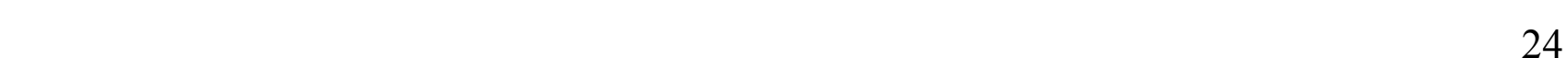

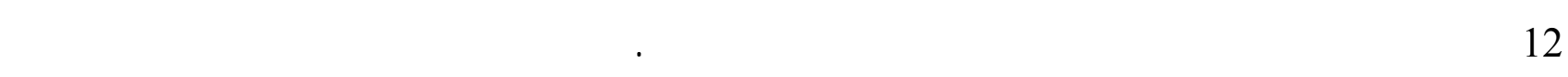

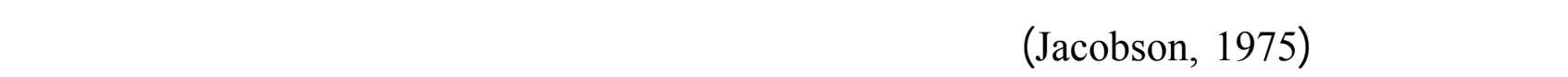
المستهوفة. 
الجدول 3: تباين التأثير القانل للمستخلصت النباتية في الطور الرابـ ع ليرق ـات البع -وض Culex pipiens المستهفة ويرقلت الهلموش Chironomus ninevah غير المستهذفة.

\begin{tabular}{|c|c|c|c|c|c|c|c|c|}
\hline \multirow{2}{*}{ لالنحدار } & \multirow{2}{*}{ قيمة F } & \multicolumn{2}{|c|}{ قيمة Lc50 (جفم) } & \multicolumn{2}{|c|}{ الفل ونسبته ليرقلت } & \multirow{2}{*}{ فترة } & \multirow{2}{*}{ 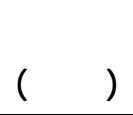 } & \multirow[t]{2}{*}{ المستخلص النبالي } \\
\hline & & للهالموث & للجوضن & الهاdوث & البعوضن & & & \\
\hline \multirow{8}{*}{ 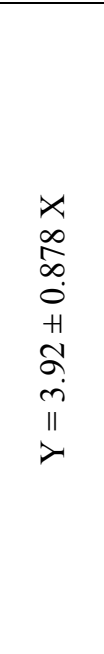 } & \multirow{8}{*}{$2158.3^{* *}$} & \multirow{4}{*}{ غيرمؤثر } & \multirow{4}{*}{27.0} & $\begin{array}{c}0.0 \pm 0.0 \mathrm{e} \\
(0.0)\end{array}$ & $\begin{array}{c}6.0 \pm 1.0 \mathrm{~d} \\
(24.0)\end{array}$ & \multirow{4}{*}{ 12ساعة } & 20 & \multirow{8}{*}{$\begin{array}{c}\text { الرمث } \\
\text { H. salicornica }\end{array}$} \\
\hline & & & & $\begin{array}{c}0.0 \pm 0.0 \mathrm{e} \\
(0.0)\end{array}$ & $\begin{array}{c}25.0 \pm 0.0 \mathrm{a} \\
(100)\end{array}$ & & 40 & \\
\hline & & & & $\begin{array}{c}0.0 \pm 0.0 \mathrm{e} \\
(0.0)\end{array}$ & $\begin{array}{c}25.0 \pm 0.0 \mathrm{a} \\
(100)\end{array}$ & & 60 & \\
\hline & & & & $\begin{array}{c}0.0 \pm 0.0 \mathrm{e} \\
(0.0)\end{array}$ & $\begin{array}{c}25.0 \pm 0.0 \mathrm{a} \\
(100)\end{array}$ & & 80 & \\
\hline & & \multirow{4}{*}{41.0} & \multirow{4}{*}{21.0} & $\begin{array}{c}0.0 \pm 0.0 \mathrm{e} \\
(0.0)\end{array}$ & $\begin{array}{c}11.0 \pm 1.0 \mathrm{c} \\
(44.0)\end{array}$ & \multirow{4}{*}{ 24ساعة } & 20 & \\
\hline & & & & $\begin{array}{c}12.0 \pm 1.0 \mathrm{~b} \\
(48.0)\end{array}$ & $\begin{array}{c}25.0 \pm 0.0 \mathrm{a} \\
(100)\end{array}$ & & 40 & \\
\hline & & & & $\begin{array}{c}25.0 \pm 0.0 \mathrm{a} \\
(100)\end{array}$ & $\begin{array}{c}25.0 \pm 0.0 \mathrm{a} \\
(100)\end{array}$ & & 60 & \\
\hline & & & & $\begin{array}{c}25 \pm 0.0 \mathrm{a} \\
(100)\end{array}$ & $\begin{array}{c}25.0 \pm 0.0 \mathrm{a} \\
(100)\end{array}$ & & 80 & \\
\hline \multirow{8}{*}{ 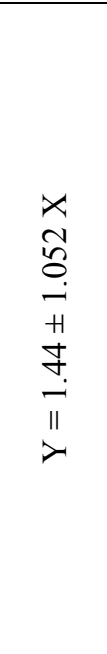 } & \multirow{8}{*}{$390.2^{* *}$} & \multirow{4}{*}{ غيرمؤثر } & \multirow{4}{*}{27.0} & $\begin{array}{c}0.0 \pm 0.0 \mathrm{~d} \\
(0.0)\end{array}$ & $\begin{array}{c}5.0 \pm 1.0 \mathrm{c} \\
(20.0)\end{array}$ & \multirow{4}{*}{ 12ساعة } & 20 & \multirow{8}{*}{$\begin{array}{l}\text { الطملة البرية } \\
\text { S. carolinense }\end{array}$} \\
\hline & & & & $\begin{array}{c}0.0 \pm 0.0 \mathrm{~d} \\
(0.0)\end{array}$ & $\begin{array}{c}25.0 \pm 0.0 \mathrm{a} \\
(100)\end{array}$ & & 40 & \\
\hline & & & & $\begin{array}{c}0.0 \pm 0.0 \mathrm{~d} \\
(0.0)\end{array}$ & $\begin{array}{c}25.0 \pm 0.0 \mathrm{a} \\
(100)\end{array}$ & & 60 & \\
\hline & & & & $\begin{array}{c}0.0 \pm 0.0 \mathrm{~d} \\
(0.0)\end{array}$ & $\begin{array}{c}25.0 \pm 0.0 \mathrm{a} \\
(100)\end{array}$ & & 80 & \\
\hline & & \multirow{4}{*}{100.0} & \multirow{4}{*}{26.0} & $\begin{array}{c}4.3 \pm 0.6 \mathrm{c} \\
(17.3)\end{array}$ & $\begin{array}{c}4.7 \pm 1.0 \mathrm{c} \\
(18.7)\end{array}$ & \multirow{4}{*}{ 24ساعة } & 20 & \\
\hline & & & & $\begin{array}{c}5.3 \pm 0.6 \mathrm{~b} \\
(21.3)\end{array}$ & $\begin{array}{c}25.0 \pm 0.0 \mathrm{a} \\
(100)\end{array}$ & & 40 & \\
\hline & & & & $\begin{array}{c}12.7 \pm 1.1 \mathrm{~b} \\
(50.3)\end{array}$ & $\begin{array}{c}25.0 \pm 0.0 \\
(100)\end{array}$ & & 60 & \\
\hline & & & & $\begin{array}{c}25.0 \pm 0.0 \mathrm{a} \\
(100)\end{array}$ & $\begin{array}{c}25.0 \pm 0.0 \mathrm{a} \\
(100)\end{array}$ & & 80 & \\
\hline \multirow{8}{*}{ 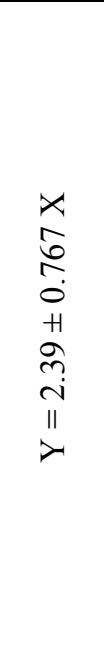 } & \multirow{8}{*}{$3788.4^{* *}$} & \multirow{4}{*}{ غيرمؤثر } & \multirow{4}{*}{20.0} & $\begin{array}{c}0.0 \pm 0.0 \mathrm{~d} \\
(0.0) \\
\end{array}$ & $\begin{array}{c}25.0 \pm 0.0 \mathrm{a} \\
(100)\end{array}$ & \multirow{4}{*}{ 12ساعة } & 20 & \multirow{8}{*}{$\begin{array}{c}\text { الزيزفون } \\
\text { T. chordate }\end{array}$} \\
\hline & & & & $\begin{array}{c}0.0 \pm 0.0 \mathrm{~d} \\
(0.0)\end{array}$ & $\begin{array}{c}25.0 \pm 0.0 \mathrm{a} \\
(100)\end{array}$ & & 40 & \\
\hline & & & & $\begin{array}{c}0.0 \pm 0.0 \mathrm{~d} \\
(0.0)\end{array}$ & $\begin{array}{c}25.0 \pm 0.0 \mathrm{a} \\
(100)\end{array}$ & & 60 & \\
\hline & & & & $\begin{array}{c}0.0 \pm 0.0 \mathrm{~d} \\
(0.0)\end{array}$ & $\begin{array}{c}25.0 \pm 0.0 \mathrm{a} \\
(100)\end{array}$ & & 80 & \\
\hline & & \multirow{4}{*}{200.0} & & $\begin{array}{c}0.0 \pm 0.0 \mathrm{~d} \\
(0.0)\end{array}$ & $\begin{array}{c}25.0 \pm 0.0 \mathrm{a} \\
(100)\end{array}$ & & 20 & \\
\hline & & & 200 & $\begin{array}{c}0.0 \pm 0.0 \mathrm{~d} \\
(0.0)\end{array}$ & $\begin{array}{c}25.0 \pm 0.0 \mathrm{a} \\
(100)\end{array}$ & . & 40 & \\
\hline & & & 20.0 & $\begin{array}{c}2.0 \pm 1.0 \mathrm{c} \\
(8.0)\end{array}$ & $\begin{array}{c}25.0 \pm 0.0 \mathrm{a} \\
(100)\end{array}$ & acluz4 & 60 & \\
\hline & & & & $\begin{array}{c}4.0 \pm 1.0 \mathrm{~b} \\
(16.0)\end{array}$ & $\begin{array}{c}25.0 \pm 0.0 \mathrm{a} \\
(100)\end{array}$ & & 80 & \\
\hline
\end{tabular}

لا يوجد فرق معنوي للأرقلم التي تحمل أرقلماً مشابهة في المريع (block) الولحد لأي فترة تعريض عند مستوى الاحتمالية 0.05. 
التأثير الحيوي امستخلص أربع نباتتا في طٔطوار.
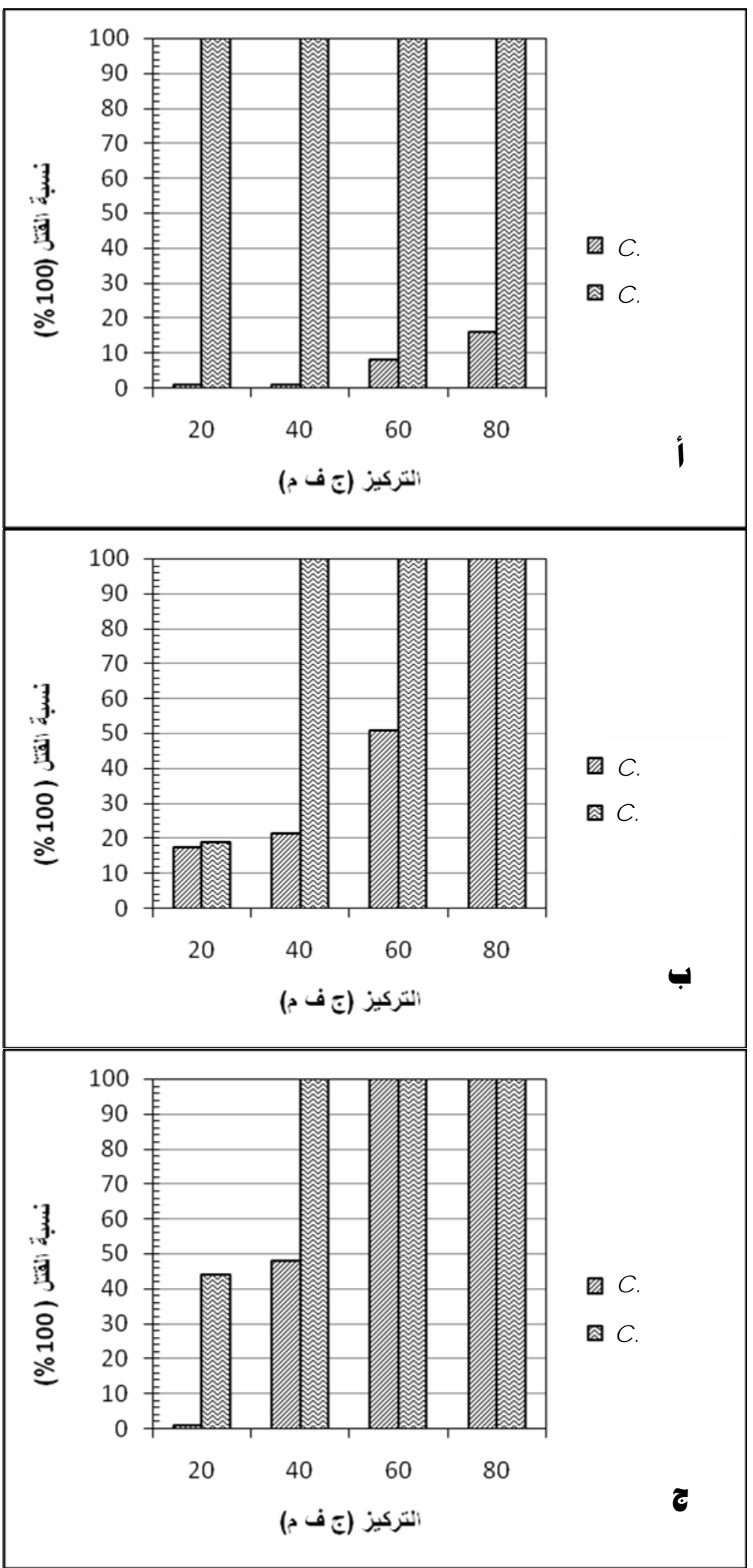

الشكل 1 : التأثير التمايزي للمستخلصت النباتية ف ي يرق لت الط -ور الرالب ع للبع -وض Culex pipiens والهلموش Chironomus ninevah بعد 24ساعة من التعريض. المستخلصك: أ. الزيزفون Titia chordata 


\section{شكر وفقير}

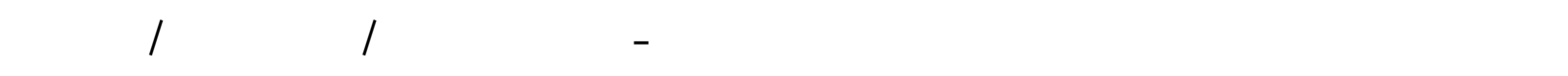
الموصل وذك لمساعته في تصنف عشرة الهلموش، Chironomus ninevah وآرائه القيمة. أتمن له دوله الصحة والتوفيق.

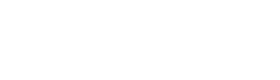

الموسوي، علي هسين عيسى (1987). علم تصنيف النبل، مديرية دار الكتب للطباء ـة والذ شرر، جلمع ـة الموصل، ص 192 -195.

\section{المصادر الأجنبية}

AL- Ahmadi, A. Z.; Salem, M. M. (1995). "Entomofauna of Saudi Arabia", Vol. 2. Checklist of phytophagous insects. King Saud Univ. Acad. Pub. Press, 187p.

Alouani, A.; Rehimi, N.; Soltani, N. (2009). Larvicidal activity of a neem tree extract (Azadirachtin) against mosquito larvae in the Republic of Algeria, Jordan J. Biol. Sc.,2(1), 15-22.

AL-Sharook, Z.; Balan, K.; Jiang, Y.; Rembold, H. (1991). Insect growth regulation from Two tropical meliaceae. Effect of crude seed extracts on mosquito larvae. J. Appl. Entomol., 111, 425-430.

Armitage , A.D. (1995). "Chironomidae as Food. In the Chironomidae: Biology and Ecology of Non-Bitting Midges", eds, P.D. Armitage, P.S. Cranston and L.C.V. Pinder, Chapman and Hall, London. pp. 423-435 .

Cranston, P.S. (1995). "The Chironomidae - The Biology and Ecology of Non- Bitting Midges". Chapman and Hall, London, Uk, 572p.

Dharmagadda, V.S.; Naik, S. N., Mittal, P. K.; Vasudevan, P. (2005). Larvicidal activity of Tayetes pattula essential oil against three mosquito species. Biosource Technol., 96, 1235-1240.

El-Hag, E. A.; Abd-El-Rahman, O.; EL-nadi, H.; Zaitoon, A. A.(2001). Effects of methanolic extracts of neem seeds on egg hatchability and larval development of Culex pipiens mosquitoes. Indian Vet. J.,78, 199-201.

Gayar, F. H.; Shazli, A. Y.; Abbassy, M. A. (1971). Toxicity of Euphorbia peplus L. (Euphorbaceae) to insects. J. Applied Entomol.,68(1-4), 63-65.

Hardin, J.W.; Arena, J.M. (1975). Human Poisoning From Native and Cultivated Plants. Duck Univ, press, 194p.

Jacobson, M. (1975). Insecticides from plants: a review of the literature, agriculture handbook. Washington, U. S. Dep. Agriculture, V. 138, 1954- 1971.

Jang, Y. S.; Kim, M.K.; Ahn, Y. J.; Lee, M. S. (2002). Larvicidal activity of Brazilian plants against Aedes aegypti and Culex pipiens Pallens (Diptera: Culicidae). Agric Chem.Biotechnol.44, 23-26.

Khater, H. F.; Shalaby, A.A. (2008). Potential of biological active plant oils to control mosquito larvae (Culex pipiens, Diptera: Culecidae) from an Egyptian locality, Rev.Inst.Med. Trop. S. Poulo, 50(2), 107-112. 
Kutas, J.; Nedasy, M.; Garf, L.; Asboth, B.(2003). Antifeedant effects of several substances on some phytophagous insect species. Zbornikpredovanj in referctov, 6, 239-243.

Makkar, H. P. S.; Sidduraju, P.; Becker, K. (2007). Plant secondary metabolites, Humonapress, Inc. 130.

Mohan, L.; Sharma, P. ;Srivastava, C. N. (2010). Combination of larvicidal action of Solanum xanthocarpum and certain insecticides against filarial vector, Culex quinqvefasciatus (Say). Southeast Asian J. Trop. Med Public Health, 41(2), 311319.

Rechinger, K. H. (1964). Flora of Lowland of Iraq. Verlag Von J. Cramer, 746.

Risk, A. M.; El-Gehazaly, G. A. (1995)." Medical and Poisonous Plants of Qatar". The Doha Modern printing press Ltd, 306p.

Shalaby, A. A.; AL-llam, K. A. M.; Moustafa, A. A. (1997). Toxicity evaluation of plant extracts on their combination with sythatic pyrethroid "cyphenothrin" against larvae of Culex pipiens (Diptera: Culicidac). Egyp. J. Med. Sci., 18, 199-212.

Siragniname, N.; Kalyanasundrama, M. (2004). Laboratory Evalutionof the methanolic extract Atlantia monophylla (Family: Rutaceae) against immature stages of mosquitoes and non-target organisms, Mon. Inst. OswaldoCruze,99, 115-118.

Sukumar, K.; perich, M. J. ; Boobar, L. R. (1999). Botanical derivatives in mosquito control- a review. J. Am. Mosq. Cont.Assoc.,7:210-231.

Sun, L.; Dong, H.; Geuo, C. (2006). Larvicidal activity of extracts of Ginkobiloba exocarp for three different strains of Culex pipiens Pallens. J. Med. Entomol., 43: 261-188.

Tidwed, J. H.; Schulmeister, C. M.; Coyle, S. (1997). Growth, survival and biochemical composition of freshwater under controlled conditions. J. the world Aquaculture Society, 28(2), 123-132.

Wais, A.W.; Wolfram, B.G.; Aver, B.; Mickschi, E.; Hain, A. (1980). Feeding habite of two introduced fish species (Leponis gibbosus and pseudoraspora parva in Neusiedler See (Australia) with special references to chironomid larvae (Dip. :Chironomidae). Hydrobiolgyia, 408/409, 123-129.

World Health Organization (WHO). (2005). Guidelines for laboratory and field testing of mosquito larvicides. WHO/ CDs/ WHOPES/GCDPP/ 2005.13.

Zayed, A. B.; Szumlas, D.E.; Hanafi, A. (2006). Use of bioassay and microplate assay to detect and measure insecticide resistance in field populations of Culex pipiens from filariasis endemic area of Egypt. J. Amer. Mosq. Contr. Ass.,22, 473-482. 